THE HEAVENS IN MARCH.

$S$ the year advances, there is a temporary break in the series of cometary phenomena which make the first half of 1910 so noteworthy. Inness's comet, which was so fine an object at the end of January, came almost to

rest among the stars early in February-moving almost straight away from the earth and rapidly losing bright. ness. By this time it is unobservable, behind the sun and some distance north of the ecliptic. In another month or so it should be seen in the morning sky, but only with telescopic aid.

The determination of its orbit has apparently presented unusual uifficulties. In order to calculate a comet's orbit, we must first have accurate observations of its position. Such observations are usually made by measuring with the telescope the distance and direction of the comet (or equivalent quantities) from some star whose place in the sky is known from previous observations, taking care to note the time at which these measures were made. In the present case the comet was at first visible only in daylight when no suitable "comparison stars" could be seen. Its position in the sky had therefore to be found by means of the readings of the readings of the graduated do the telescope; and these are far inferior in accuracy to measures of the former kind.

It is not therefore surprising that the preliminary orbits calculated from these rough observations differ widely among themselves. Whe $\mathrm{n}$ th comet reappears in the morning sky, and can be accurately observed, the comparison of the results with the few accurate observations made in January and February will settle the question.

Halley's comet is also out of sight for the present-behind the sun, or nearly so, and about 175 million miles away. It may still be observed telescopically, low in the west in the early evening, for a few days early in the month; but it cannot be seen in the morning sky until April is well begun, when it will ' reappear, much nearer us, and probably far brighter than when it vanished in the twilight.

We must therefore for the time being turn our attention mainly to the stars, and among them we will find much to occupy us while we wait for the comet to reappear. Let us begin right overhead with the constellation Gemini. Its two principal stars, Castor and Pollux, identify it at once, for nowhere in our skies are two equally bright stars as near neighbors. The roughiy parallel lines of stars which run south west from these and terminate in the stars $\gamma$ and are also easy to identify if once learned.

Castor itself is a fine object with even a small telescope, showing double with a power of fifty diameters. The two components, one about twice as bright as the other, revolve about one another with a period of perhaps 400 years. The faint star which lies about twelve times as far from them as they are from one anothe is moving with them among the other stars, and is probably also in very slow revolution about them, but, if so, must take more than ten thousand years to complete $r$. single circuit. The star. $\delta$ is also a fine double. Close by to the eastward is Cancer, with no bright stars, but one interesting cluster, visible clearly to the naked eye, and resolved into its separate star by a field glass. This spot of light, known as Praesepe (the Beehive), is marked on the map.

Auriga, which lies in the Milky. Way, northwest of the zenith, is one of the finest constellations in the sky. The figure of the charioteer kneeling in his chariot forms our initial letter. The most northerly bright star of the constellation, $\delta$, is in his head, $\beta$ is in his right Capella (a) marks hold in his left arm, while the three smaller stars just below it are called the kids-an armful indeed! Farther south $\theta$ is in his right knee, and $z$ in his left foot, uncomfortably near the tip of one of the Bull's horns ( $\beta$ Tauri).

Capella is, next to Sirius, the brightest star in the part of the skies which we can see-according to the data of the Nautical Almanac. Arcturus and Vega are however very nearly its equal in brightness, and the servers, whose eyes were unequally sensitive to light of different colors, might easily disagree as to their relative rank. Well down in the west are Orion and his neighbors, Canis Major on the left and Taurus on the right Mars passes through the latter constella. thion during the month and fairly rivals Aldebaran in color and brightness. Perseus and Andromeda are in the northwest-the latter partly set-and Cassiopeia

Due south we see Procyon, with the few faint at tendants which are supposed to resemble the Little Dog, high up near the meridian, and part of the great constellation Argo low on the horizon. Farther east, three stars differ so widely in color that different obfarther to the left.

right of the pole; Ursa Minor, inclosed within its sweep; and Cepheus, low in the north, complete our survey.

\section{THE PLANeTs.}

Mercury is morning star all through March, but being south of the sun, is not well observable in these latitudes. He may perhaps be seen early in the month, but only with difficulty, as he rises but three-quarters of an hour before the sun.

Venus is likewise a morning star; and, unlike Mercury, is very conspicuous.

She reaches her greatest brightness on the 18th. At this time she appears, with a magnifying power of fifty diameters, just as the crescent moon some five days old does to the naked eye, except that her surface is free from perceptible markings, and uniformly white. She rises about 4 A. M. and is the principal ornament of the morning sky. Indeed, there is no difficulty about seeing her in broad daylight-if the sky is really clear-except that of knowing where to look for her. On the morning of the 16th the crescent moon will be a good guide, as Venus will lie about five or six degrees nearer the sun, and about two degrees above the line joining them.

Mars is still an evening star, but is steadily losing brightness. His distance from us increases from 145 to 170 million miles during the month, and he moves eastward among the stars through Taurus, keeping well ahead of the sun, so that he remains in sight till after $11 \mathrm{P}$. M.

Jupiter is in opposition on the 31st, and is visible all night long. $\mathrm{He}$ is a splendid object to the naked eye, and a fascinat ing one in even the smallest telescope. His four large satellites can be seen without difficulty, unless indeed $\mathrm{some}$ of $\mathrm{them}$ should be behind or in front of the planet. In the latter case their shadows can be seen, as black dots on Jupiter's surface, with more powerful in struments, and afford one of the most interesting of celestial spectacles as they transit across his disk. The elliptical form of the planet due to his rapid rotation, and the dark belts which cross his disk parallel to his equator, can also be seen with a small in strument. The four other satellites, discovered in recent years, are observable only with a few of the largest telescopes.

Saturn is an evening star, setting about $8 \mathrm{P}$. M. in the middle of the month. Uranus is in $\mathrm{Sa}$ gittarius, $r$ is ing about $3: 30$ A. M. at the same date. Neptune is well observable in Gemini, his position on the 15 th being R. A. 7 h. 11 m. 35 s., Dec. $21 \mathrm{deg} .44 \mathrm{~min}$. north, and

\section{NIGHT SKY: FEBRUARY AND MAREH}

on a level with Procyon, a small but conspicuous group marks the head of the great serpent Hydra, whose body may be followed, past the lonely red star Alphard, down to the southeastern horizon. To the left are Corvus and Crater, and then Virgo, made unusually brilliant by the presence of Jupiter, which is just below the notable double star $\gamma$. Higher up is Leo, one of the finer constellations, recognized at once by the "Sickle," at the end of whose handle is the first-magnitude star Regulus.

Farther west, near the horizon, Arcturus shines brilliantly, far surpassing his neighbors in Boötes Above and on the left is the familiar and gigantic form of the Great Bear. Within the curve of the Bear's tail (the Dipper handle) are the Hunting Dogs which pursue her on an endless chase around the pole. They have but a single bright star, but this is a fine double, worth looking at if one has a telescope magnifying twenty times or more.

The group of small stars known as Berenice's Hair, to the southward, is a good example of a star cluster so coarse that it can be resolved by the naked eye but yet composed of stars faint enough, and nea enough together, to produce almost the impression of a nebula at a hasty glance, while closer scrutiny shows the individual components. Draco, coming up on the
At 9 o'clock: 8 sis. 8.

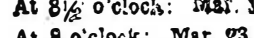
An o'clock: slow. To identify him, howhis apparent motion very slow. To idar map or a tele ever, one needs either a detald disk; that is, six inches or so in aperture.

The moon is in her last quarter at $3 \mathrm{~A}$. M. on the th, is. new at $7 \mathrm{~A}$. M. on the 11 th, in her first quarter at $10 \mathrm{P}$. M. on the $17 \mathrm{th}$, and is full at $3 \mathrm{P}$. M. on the 25th. She is nearest us on the 12 th, and farthest of on the 28th. In her circuit around the sky she passe near Uranus on the 7th, Venus on the 8th, Mercury on the 9th, Saturn on the 13th, Mars on the 16th Neptune on the 19th, and Jupiter early on the morning of the 26 th-only the last conjunction being at all close.

At 7 A. M. on March 21st the sun crosses the celestial equator, passing over the point in the heaven called the vernal equinox, or "first point of Aries," and in almanac language "Spring commences."

Princeton University Observatory.

Gilding Glass or Porcelain.-Prepare a mixture of 900 parts of lavender oil, 100 parts of gold chloride, 5 parts of bisnuth subnitrate, and 50 parts of chrome soap. After application, leave the mass to dry and bake the article in a muffle furnace. A brilliant gilding will be produced without further treatme日t. 